\title{
INFECCIÓN POR BACTERIAS DE VÍAS URINARIAS EN MUJERES TRATADAS CON CATÉTER URETRAL Y RESISTENCIA BACTERIANA A ANTIBIÓTICOS
}

\section{INFECTION BY BACTERIA OF DEVELOPING URINARY TRACT IN WOMEN TREATED WITH URETHRAL CATHETER AND BACTERIAL RESISTANCE AN ANTIBIOTICS}

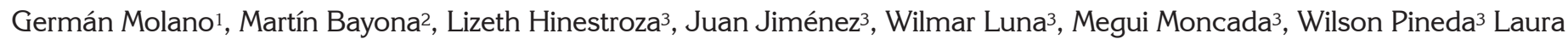
Plazas 3 , Carlelys Ríos 3 , Hadelly Runza ${ }^{3}$

1 M.D. Especialista en Epidemiología. Docente Facultad de Medicina Universidad de Ciencias Aplicadas y Ambientales. U.D.C.A. 2 Bacteriólogo, Especialista, M.Sc. Microbiología. Facultad de Medicina, Universidad de Ciencias Aplicadas y Ambientales U.D.C.A. Calle 222 No. 55-37, Bogotá, D.C. e-mail: mabayona@udca.edu.co: autor para correspondencia. ${ }^{3}$ Estudiantes, Facultad de Medicina. Universidad de Ciencias Aplicadas y Ambientales U.D.C.A.

Rev. U.D.C.A Act. \& Div. Cient. 15(1): 27 - 34, 2012

\section{RESUMEN}

A pesar de los protocolos orientados a la prevención de infecciones asociadas con catéter urinario en hospitales de III y IV nivel en la ciudad de Bogotá y el municipio de Soacha, éstas representan un incremento en tiempo y en costo de estadía del paciente. Se realizó un estudio descriptivo, serie de casos, cuyo objetivo fue determinar la frecuencia y la sensibilidad de los antibióticos frente a los microorganismos asociados con infección de vías urinarias, en mujeres de 40 a 80 años, quienes requirieron de cateterismo uretral, en unidades de cuidados intensivos, de cuatro hospitales de III y IV nivel de complejidad. Se evaluaron las historias clínicas de las pacientes, quienes cumplieron con requisitos de inclusión en el periodo comprendido entre enero y diciembre de 2010, teniendo en cuenta las variables identificadas y consignadas en el instrumento correspondiente, como fueron: hospital, edad, tiempo de estancia, tiempo de duración del catéter, urocultivo y resultado del antibiograma. Para tabular y analizar los datos, se emplearon el programa Microsoft Excel y el estadístico SPSS - 15, para posteriormente recodificar las variables y generar medidas de resumen, medidas de dispersión, frecuencias absolutas, tablas y gráficas. Escherichia coli representó el 39\% del aislamiento total en los cuatro hospitales, seguido por Klebsiella pneumoniae (14,9\%), Proteus mirabilis (8,5\%), Klebsiella sp. y Pseudomonas aeruginosa (5,7\%). Se observó resistencia de ampicilina en 36,8\%, de cefalexina (30,9\%), de tetraciclina $(29,4 \%)$ y de nitrofurantoína (23,5\%). La institución 1 reportó el mayor número de casos asociados a infección urinaria y se identificó el mayor intervalo de edad, correspondiente a 60 a 69 años.

Palabras clave: Bacterias, catéter, infección urinaria, resistencia bacteriana, salud pública.

\section{SUMMARY}

Despite the protocols aimed at preventing infections associated with urinary catheter in hospitals of III and IV level in Bogotá and Soacha, they represent an increase in time and cost of patients stay. A descriptive study was carried out number of cases, which the objective to determine the frequency and sensitivity of antibiotics against microorganisms associated with urinary tract infection in women aged 40 to 80 requiring urethral catheterization in intensive care of four hospitals in III and IV level of complexity. The clinical histories of patients who met the established requirements for inclusion in the period between January and December 2010 were assessed, taking into account the predetermined variables included in the corresponding instrument as were: hospital, age, length of permanence, duration of catheter, urine culture and result of the antibiogram. To tabulate and analyze the data Microsoft Excel and SPSS statistical - 15 program were used, in order to subsequently recode variables and to generate sum up and dispersion measures, absolute frequencies, tables and graphs. Escherichia coli accounted for $39 \%$ of the total isolation in four hospitals, followed by Klebsiella pneumoniae (14.9), Proteus mirabilis (8.5), Klebsiella sp. and Pseudomo- 
nas aeruginosa (5.7). Ampicillin resistance was observed in $36.8 \%$, Cefalexin (30.9\%), tetracycline (29.4\%) and nitrofurantoin (23.5\%). Institution 1 reported the highest number of cases associated with urinary tract infection; as most vulnerable age range women 60-69 years old, were identified.

Key words: Bacteria, catheter, urinary tract infection, bacterial resistance, public health.

\section{INTRODUCCIÓN}

La infección de vías urinarias es definida como la existencia de microorganismos patógenos en el tracto urinario, con o sin presencia de síntomas específicos y, en el caso de presentar síntomas, se evidencia urgencia miccional, disuria, tenesmo, dolor supra púbico y fiebre (Chung et al. 2008). Estas infecciones cuando ocurren en un ambiente hospitalario, se evidencian en pacientes que requieren la utilización de un catéter uretral, a la que se le suman una serie de factores que ocasionan, finalmente, vulnerabilidad por parte del mismo, para desarrollar la infección, tales como: errores en la técnica de colocación, deficiencia durante los procesos de asepsia y antisepsia del personal de salud y del lugar donde se va a realizar el procedimiento, inadecuada práctica por parte del médico y enfermedades de base (diabetes mellitus e insuficiencia renal crónica), entre otros factores (Rao et al. 2011; Pavanello et al. 2009; Álvarez et al. 2007).

La medicina moderna depende de agentes químicos empleados para el tratamiento de enfermedades. Su eficacia se asocia con muchos factores, entre ellos la ruta de administración, la localización de la infección, la presencia de sustancias que interfieren con el fármaco, la concentración de fármacos en el cuerpo, la naturaleza del patógeno, la existencia de alergias y la resistencia de los microorganismos a los fármacos; éste último aspecto, con un creciente número y variedad de patógenos, constituyéndose así en un serio problema de salud pública (Willey et al. 2009). Hoy en día, es claro que el tratamiento de los procesos infecciosos se basa en el diagnóstico clínico, de tal manera que, la elección del antimicrobiano que se vaya a emplear, se realiza de forma empírica o fundamentada en diversos estudios. La prescripción de antibióticos, se realiza, en muchas ocasiones, antes de conocer los resultados del antibiograma y es frecuente que para su elaboración no se tenga en cuenta el perfil epidemiológico de la localidad, ni los agentes etiológicos más prevalentes en el medio, ni el perfil de sensibilidad de los mismos (Xie et al. 2011; Ochoa et al. 2005).

Las infecciones asociadas al cuidado de la salud en pacientes ingresados a la unidad de cuidados intensivos, se han convertido en un inconveniente para los hospitales, ya que se han vuelto un problema costo-efectivo para éstos, aumen- tando el precio de la hospitalización del paciente; de igual manera, con el ingreso de pacientes a la Unidad de Cuidados Intensivos (UCI), se pretende realizar una monitorización rigurosa de su evolución y, de esta manera, poder estar alerta sobre cualquier cambio que pueda comprometer el estado de salud de los mismos (Amin et al. 2009; Álvarez et al. 2007; Horan et al. 2008).

Es sabido por el personal médico y hospitalario, que las infecciones asociadas al cuidado de la salud se presentan por la utilización de mecanismos de soporte para pacientes en estado crítico, como son la ventilación mecánica y catéteres, tales como los uretrales, centrales, traqueotomías y gastrostomías; debido a lo anterior, las infecciones producidas por este tipo de métodos se han convertido en un problema de salud intrahospitalaria, ya que, en algunos casos, es necesario llevar al paciente a estos procedimientos, a expensas de preservar la vida del mismo y es durante su estancia hospitalaria que son infectados por microorganismos que, a través de los años, han desarrollado mecanismos de resistencia, mucho más eficientes (Amin et al. 2009; De Oliveira et al. 2010; Rosenthal et al. 2010).

El tracto urinario representa más del $40 \%$ del total de infecciones intrahospitalarias (IIH) notificadas; la mayoría, entre el 66 y $86 \%$ ocurre después de procedimientos que implican instrumentación del tracto urinario, principalmente, por la sonda vesical o catéteres vesicales. Las infecciones del tracto urinario asociadas a catéter son causadas por una amplia variedad de patógenos, específicamente, bacterias Gram negativas, como Pseudomonas sp., Acinetobacter sp., Escherichia coli y Klebsiella sp. Es importante resaltar que los grados de resistencia pueden variar mucho de un hospital a otro, de una ciudad a otra y de una Nación a otra (Álvarez et al. 2007; Sader, 2002; Talaat et al. 2010; González Camarena et al. 2009; Echevarría et al. 2006; Eiros \& Ochoa, 2007).

Con base en lo anterior, si se tiene el conocimiento sobre los microorganismos predominantes en los hospitales objetos de estudio y sus respectivas resistencias, el uso del tratamiento adecuado contribuiría a disminuir la utilización innecesaria de antibióticos, a los cuales, se presenta mayor resistencia por parte de los microorganismos aislados y, de esta manera, ayudar a la disminución de los costos destinados al tratamiento con antibiótico.

\section{MATERIALES Y MÉTODOS}

La presente investigación es de tipo descriptivo, serie de casos realizada en Unidades de Cuidados Intensivos (UCI), de cuatro hospitales ubicados en la ciudad de Bogotá y municipio de Soacha, correspondientes al tercer y cuarto nivel de complejidad. Los hospitales se definieron como: Institución 
1, en donde se habilita para la atención de sus pacientes: una unidad de cuidado coronario y una unidad de cuidados intensivos; Institución 2, que cuenta con una unidad de cuidados intensivos; Institución 3, que tiene a su disponibilidad una unidad de cuidados intensivos e, Institución 4, que cuenta con cuatro unidades de cuidados intensivos.

La población evaluada correspondió a pacientes de género femenino, ingresadas al servicio de Unidades de Cuidados Intensivos, de cuatro hospitales, en el periodo comprendido entre enero y diciembre de 2010. Se revisaron un total de 449 historias clínicas de pacientes mujeres, entre 40 y 80 años de edad.

Criterios de inclusión: Para este estudio, se incluyeron pacientes cuya edad figuraba entre 40 y 80 años, hospitalizadas en $\mathrm{UCl}$, con inserción de catéter uretral dentro de la misma institución, en un tiempo no mayor a seis horas y aquellas a las quienes se les diagnosticó infección urinaria, durante su hospitalización. Solamente se incluyeron personas cuya historia era completa.
Se excluyeron: Pacientes con diagnóstico de anormalidad anatómica del tracto urinario de base, que ingresaron a la $\mathrm{UCI}$, remitidas de otros centros hospitalarios y que traen catéter uretral.

La técnica de recolección de datos, se realizó por medio del análisis de historias clínicas de pacientes ingresados en los servicios de Unidad de Cuidados Intensivos, en cada uno de los hospitales, objeto de estudio; las muestras de catéter urinario fueron enviadas al laboratorio de los hospitales evaluados de microbiología, para su cultivo en agar CLED, el cual, fue incubado a 37 grados Celsius, por 24 horas. La identificación del microrganismo, se realizó empleando pruebas bioquímicas, mediante el empleo del sistema API 20E. La susceptibilidad antibiótica, se efectuó mediante el método de Kirby Bauer en agar Mueller Hinton, utilizando un inóculo con turbidez de 0,5, en la escala de Macfarlánd. Teniendo en cuenta lo anterior, se definieron y se consignaron las variables en el instrumento aplicado (Cuadro1), incluyendo as-

Cuadro 1. Formato de recolección de datos en los hospitales objeto de estudio.

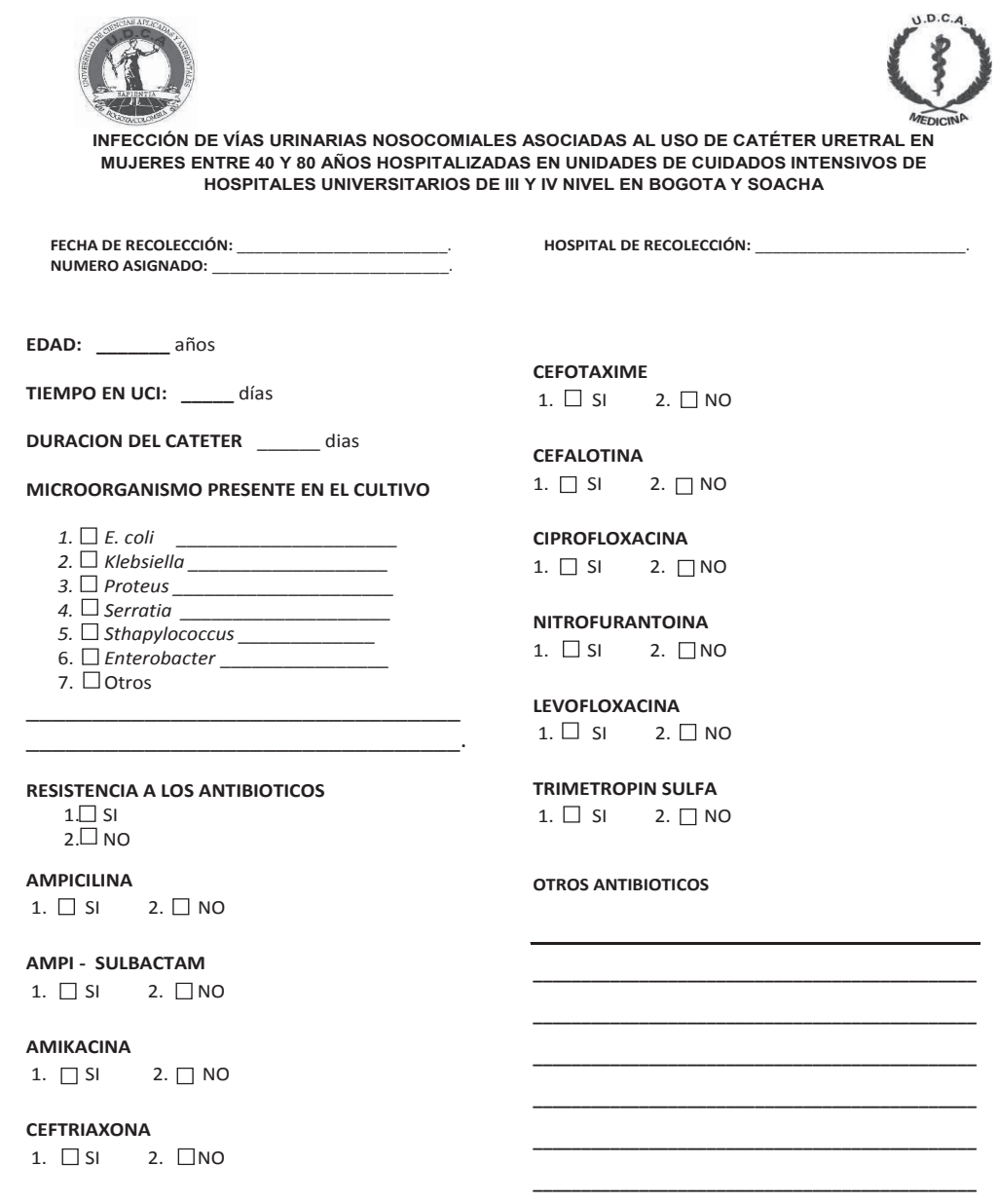


pectos como: hospital, edad, tiempo de estancia en la $\mathrm{UCI}$, tiempo de duración del catéter, microorganismos aislados en el uro-cultivo y resultado del antibiograma.

Análisis estadístico: Una vez revisadas las historias clínicas, se construyó una base de datos, empleando la hoja de cálculo de Microsoft Office Excel. Teniendo en cuenta los criterios clínicos establecidos, se formalizó la codificación de los datos, para luego efectuar un análisis uni-variado y bi-variado, utilizando el paquete estadístico SPSS (Statistical Package for the Social Sciences) versión 15.0.; posteriormente, se realizó la recodificación de las variables y la generación de medidas de resumen, medidas de dispersión, frecuencias absolutas, tablas y gráficas.

Aspectos éticos y legales: Durante la realización del presente trabajo, se buscó la obtención de la información, de tal forma, que no vulnerar los derechos de las personas, en estado de pacientes y que fueron objeto de nuestra investigación; de igual manera, no se reveló la identidad del paciente, ni las instituciones en donde se recolectaron los datos.

\section{RESULTADOS Y DISCUSIÓN}

Se revisaron un total de 449 historias clínicas de pacientes mujeres, entre 40 - 80 años de edad. Dichas historias, se vieron representadas de la siguiente manera: Institución 1: 293 historias clínicas; Institución 2: 32; Institución 3: 75 y, en la Institución cuatro: 49 historias clínicas. De las 449 historias, 141 cumplieron con los criterios de inclusión, establecidos en el presente trabajo.

Las instituciones en que más se presentaron los casos de infección urinaria correspondieron en su orden al número uno, seguida por la número cuatro y, finalmente, las números dos y tres (Figura 1).

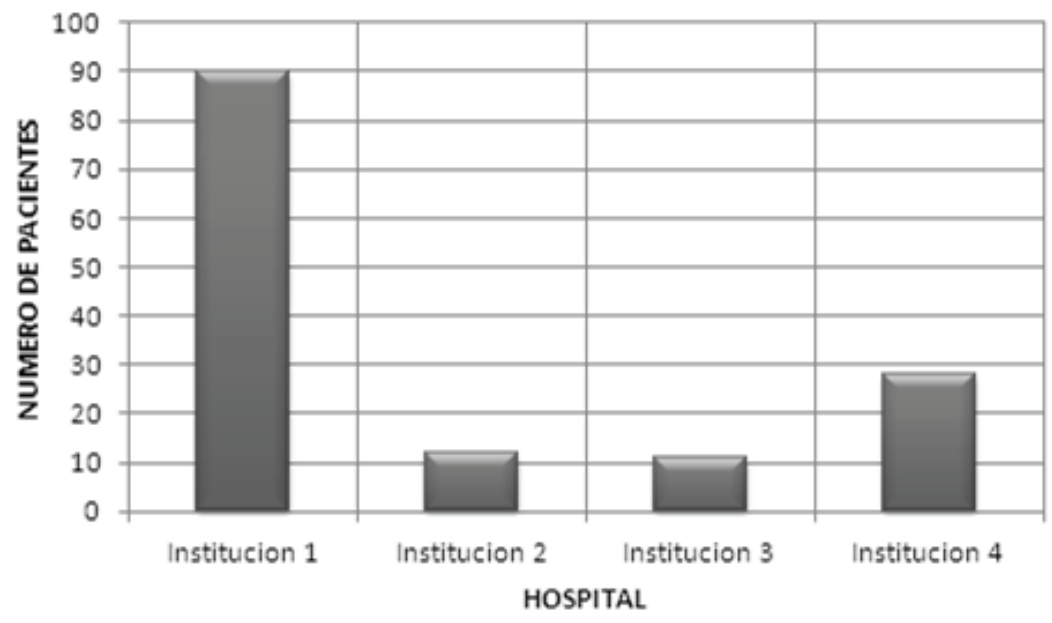

Figura 1. Distribución de la infección urinaria asociada al cuidado médico, en Unidades de cuidado intensivo por institución. Enero a diciembre de 2010.

Los microorganismos aislados con mayor frecuencia correspondieron a: E. coli (39\%); K. pneumoniae (14,9\%); P. mirabilis (12,5\%); P. aeruginosa (5,7\%) y Klebsiella spp. (5,7\%). Entre los microorganismos que menos se aislaron en los uro-cultivos fueron: K. ornithinolytica, K. ascorbata, Morganella morganii, Providencia stuartii, Edwardsiella tarda, $K$. oxytoca y Raoultella ornithinolytica, con un porcentaje menor del 3\% (Figura 2); estos resultados son similares a los hallados en el estudio realizado por Verhaz et al. (2005), quienes aislaron, a partir de uro-cultivos: $E$. coli $(31,0 \%) ; P$. aeruginosa (13,8\%); P. mirabilis (12\%); Klebsiella spp. y Enterobacter spp. (12,3\%), Enterococcus spp. (5,2\%); de igual manera, en el estudio presentado por Amin et al. (2009), en la ciudad de Ahvaz, Irán, donde se determinó que los microorganismo más frecuentes en la presentación de la infección de vías urinarias correspondieron a: E. coli (59\%), seguido por Klebsiella spp. (11,6\%); Enterobacter spp. (9,8\%) y Pseudomonas sp. (7,2\%). Nuestros datos contrastan con el estudio realizado por Mindy et al. (2010), en Singapur, donde encontraron 35 pacientes, quienes presentaron infección de vías urinarias asociada al cuidado de la salud, en los que el microorganismo más frecuente fue Candida spp. Por otra parte, en un estudio similar descrito por Álvarez et al. (2007), realizado en España, entre el 2003 al 2005, describieron que 


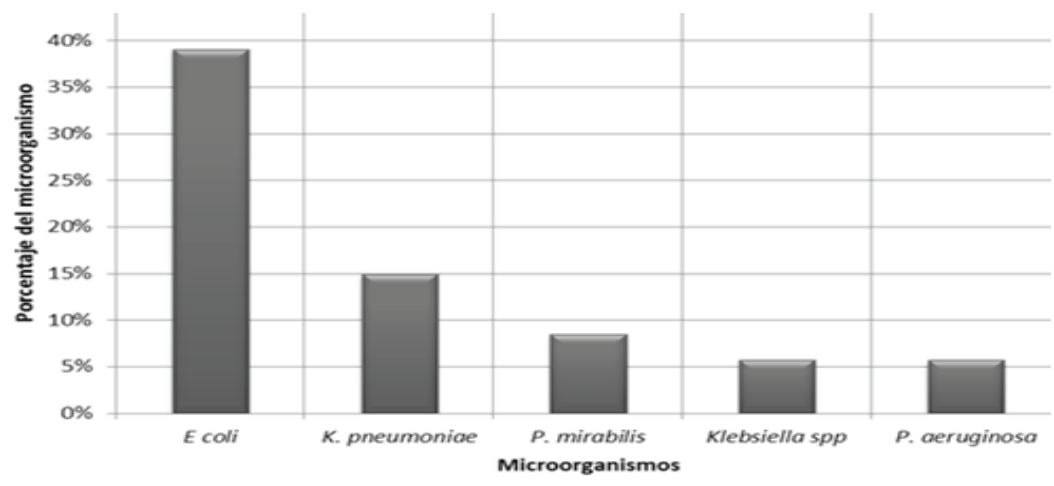

Figura 2. Porcentaje de microorganismos aislados a partir de urocultivos de pacientes femeninas hospitalizadas en unidades de cuidados intensivos de las instituciones evaluadas. Enero a Diciembre de 2010.

E. coli fue el principal microrganismo, seguido de Candida albicans y/o Candida spp.

En nuestro estudio, no se reportaron hongos tipo Candida. Podemos inferir que los resultados no son similares a lo reportado en la literatura mundial, debido a diversas condiciones socio-demográficas entre los mismos; además, debemos tener en cuenta que algunos de los estudios revelados no tienen en cuenta, únicamente, la infección de vías urinarias, ya que incluyen, en los mismos, toda infección asociada al cuidado médico en unidades de cuidados intensivos.

Con el análisis de los datos recolectados fue posible describir los microorganismos implicados en la génesis de la infección de vías urinarias de las pacientes, incluidas en el estudio (Figura 3). El microorganismo más común registrado en las historias clínicas de los cuatro hospitales fue E. coli, con un total de 55 casos, representado en el 39\%, del total de los resultados. Se determinó que el segundo lugar lo ocupó $K$. pneumoniae, con un $14,9 \%$, seguido por P. mirabilis, con $8,5 \%$, Klebsiella spp., 5,7\% y $P$. aeruginosa, 5,7\%. Según los resultados obtenidos, se evidencia que en la Institución 1 fue donde se presentaron mayor número de casos (90), que corresponden al $63,8 \%$ del total de las historias revisadas; en segundo lugar, se encuentra la Institución 4 , con 28 casos, que indica $19,9 \%$, del total de los casos, seguida por la Institución 2, con 12 casos y el 8,5\%, en último lugar, se la Institución 3, la cual, registró once casos, que representan el $7,8 \%$, del total de los casos evaluados.

Entre los antibióticos que relacionaron mayor resistencia (Figura 4), se regostran: Ampicilina, en un 36\%; Tetracicli-

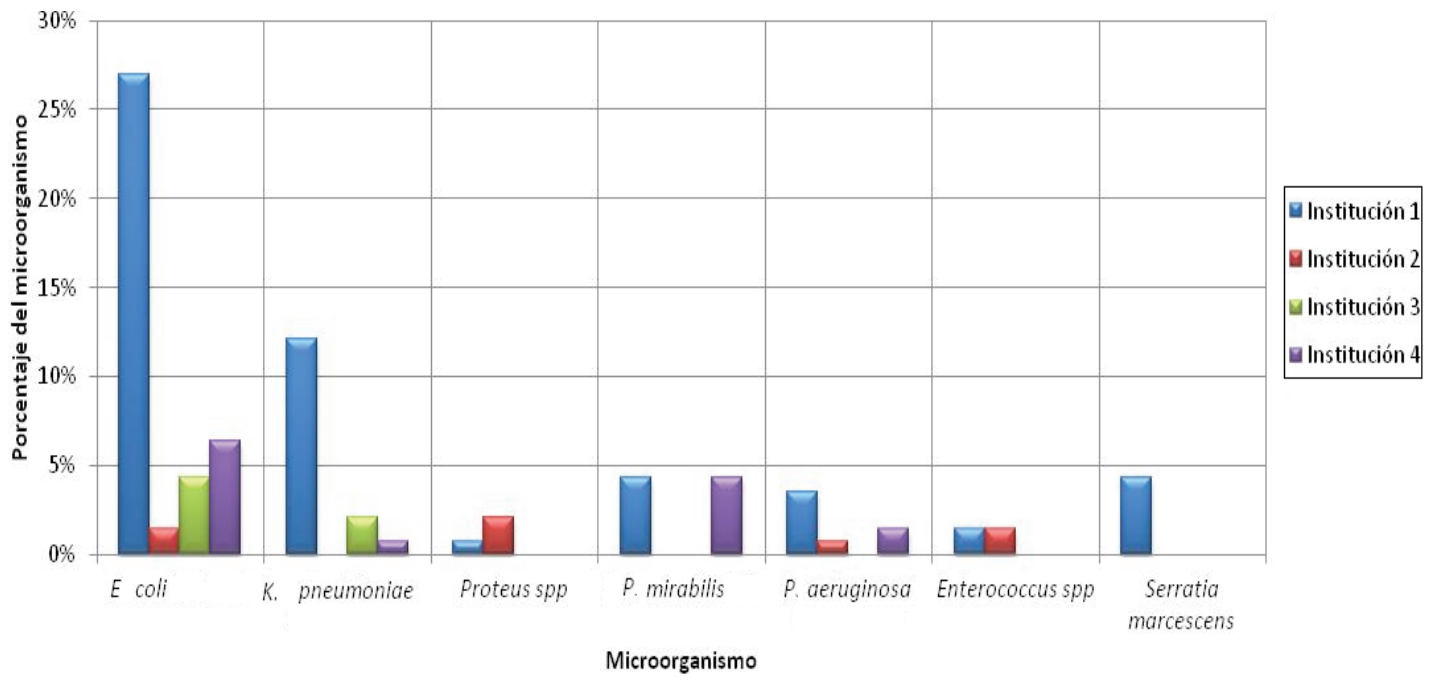

Figura 3. Porcentajes de los microorganismos más frecuentes, aislados en los cultivos, de los cuatro hospitales. 


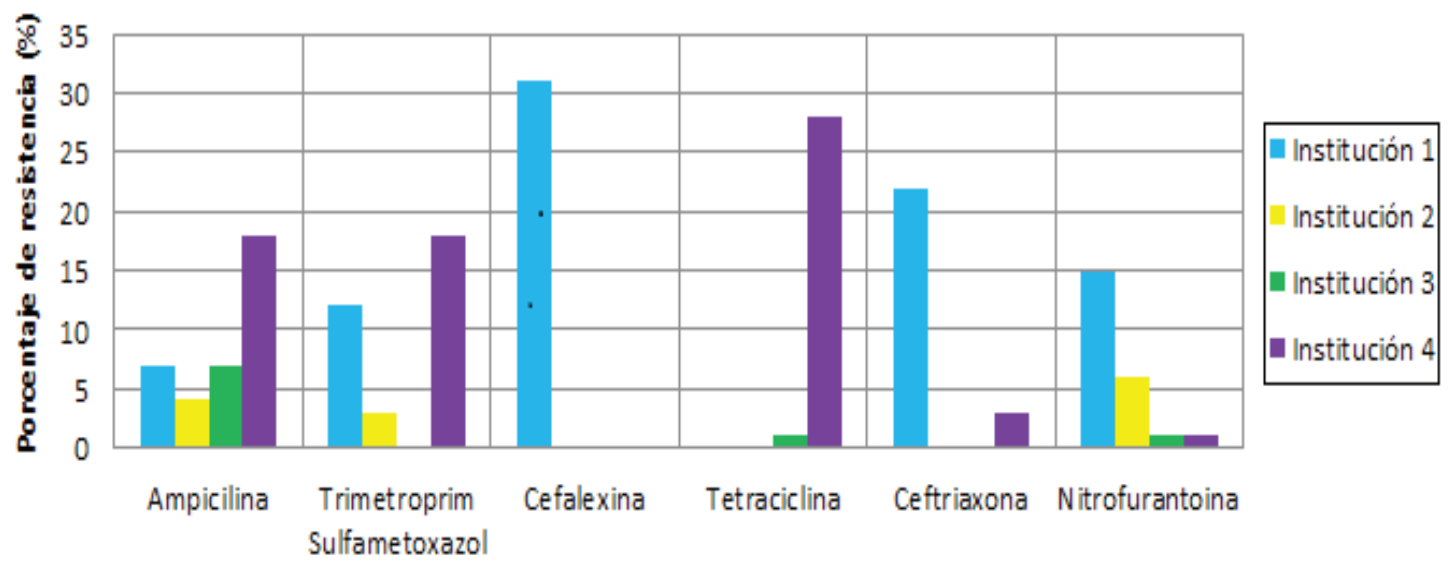

Figura 4. Porcentaje de resistencia antibiótica reportada en el antibiograma de cada institución, objeto de estudio.

na, en 29,4\%; Ceftriaxona, 25\% y Ciprofloxacina, en 25\%, al compararse con los resultados demostrados en el estudio realizado por Verhaz et al. (2005), donde evaluaron 265 pacientes hospitalizados en la UCI del Centro Clínico de Banja Luka, en el que reportan que Ampicilina fue el antibiótico que presentó la mayor resistencia, con un 64\%. Por otra parte, en el estudio llevado a cabo por Álvarez et al. (2007), en $14 \mathrm{UCl}$, en Bogotá, Colombia, donde reportan resistencia a Ciprofloxacina, en más de un $20 \%$. Las diferencias con nuestros resultados al hablar de porcentajes, se explicarían, porque las muestras tomadas para los estudios Álvarez et al. (2007) y Verhaz et al. (2005) son significativamente mayores. La aparición de microorganismos multi-resistentes, se podría explicar por el uso indiscriminado de los antibióticos, en presencia de infección bacteriana. La Organización Panamericana de Salud define la relación entre el uso de antibióticos y la propagación de microorganismos resistentes, como un factor de complicación inherente, que contribuye al aumento de la resistencia a los antimicrobianos (Salvatierra \& Benguigui, 2000; Talaat et al. 2010).

Al realizar el cruce entre las variables: microorganismos aislados frente a la resistencia a los antibióticos fue posible establecer que $E$. coli presentó resistencia a Cefalexina y Ampicilina cada uno con $12 \%$, Tetraciclina y Ampicilina Sulbactam con el 9\% respectivamente y Ciprofloxacina 7\%, para el caso de $P$. mirabilis se identificó que presentó resistencia a Tetraciclina 9\%, Ampicilina, Cefalexina y Ceftriaxona cada uno con 3\%, dichos resultados coinciden con el estudio realizado por Verhaz et al.(2005), donde también se encontró que Ampicilina, Gentamicina y Tetraciclina fueron los antibióticos, a los cuales, se presentó mayor resistencia microbiana. Lo anterior es posible relacionarlo con el aumento en el uso de dichos antibióticos, en los hospitales objeto de estudio.

El microorganismo que se registró con mayor frecuencia en todos los rangos de edad fue $E$. coli y la edad en la que se manifestaron más casos de infección urinaria fue en mujeres mayores de 65 años, destacando el rango de edad de 60-69 años, seguido por el de 70-79 años. A partir de los datos, se logró establecer que la media de la edad fue 62,5 años, dato que coincide con la investigación de Rao et al. (2011), realizada en el hospital Wenzhou, de China, mostrando un promedio de 62,7 años. similar a lo encontrado en la investigación elaborada por Chung et al. (2008), en el que halló que la presencia de la $E$. coli, en mayores de 65 años, con cateterización, era más alta que en las menores de 65 años. De igual manera, Pavanello et al. (2009) percibieron en las Unidades de Cuidado Intensivo de las instituciones de salud en Sao Paulo, Brasil, entre 2003 y 2005, que el desarrollo de la infección urinaria en pacientes mayores de 61 años, se presentó en un $73 \%$ de los casos, al igual que quienes evidenciaron datos similares, a partir de sus resultados, en 178 pacientes, con infección del tracto urinario, obteniendo que la edad media correspondió a 61 años y el microorganismo más frecuentemente aislado fue $E$. coli $(31,4 \%)$, seguido por Candida spp. (21,3\%), Klebsiella spp. (10,6\%) y Enterococcus spp. (6,9\%). Elpern et al. (2009), reportan una media de 61 años; resultados que explican las posibles comorbilidades de las pacientes en esta etapa de su vida, como lo son: enfermedades neurológicas, diabetes mellitus, inmunosupresión y enfermedades renales. También es necesario conocer el tiempo de estancia en UCl y el tiempo de cateterización, en el que se presenta, frecuentemente, la infección 
de vías urinarias; esto nos permite inferir el riesgo de una paciente a padecer la infección y poder tomar medidas para prevenir la aparición de éstas y sus posibles complicaciones. En las instituciones estudiadas, se evidenció que la infección urinaria se mostró entre los diez primeros días, en un $68 \%$ y disminuye su frecuencia, entre más días de estancia en UCI tenga, contrario a un estudio hecho por Talaat et al. (2010), llevado a cabo en unidades de cuidados intensivos (UCI), del hospital universitario de Alejandría en Egipto. Una de las conclusiones fue que una hospitalización prolongada en UCI se entendía como un factor de riesgo para desarrollar la infección urinaria; en cuanto al tiempo de cateterización, la aparición de infección urinaria también se da en los primeros diez días, en un $80,1 \%$.

Afín a un estudio logrado por Elpern et al. (2009), evidenciaron que es necesario limitar el tiempo de uso del catéter, ya que, de esta forma, se disminuye el riesgo de presentar infección urinaria. En otro estudio realizado por Pavanello et al. (2009), en Unidades de Cuidados Intensivos de las instituciones de salud en Sao Paulo, Brasil, entre 2003 y 2005, publicado en 2009 , concluyó que con un tiempo prolongado de internación en $\mathrm{UCI}$, con uso de sonda vesical (>15 días), se genera mayor asociación a infección urinaria.

Se observó que en los grupos etarios, el que presentó mayor cantidad de pacientes fue el grupo de 60-69 años de edad, seguido por el grupo cuatro, que corresponde a edades entre 70-79 años; seguido por los grupos etario dos (50-59 años) y uno (40-49 años). Finalmente, se observa que el grupo de 80 o más años fue en el que se presentó el menor número de pacientes.

Teniendo en cuenta el microorganismo aislado en el urocultivo, se estableció el vínculo entre éste y la edad de las pacientes. El microorganismo más frecuente fue $E$. coli, presentándose con mayor frecuencia en el rango de edad comprendido entre 60 a 69 años, correspondiente al 11,3\%, seguido por el rango de 50 a 59 años, con 10,6\% y 9,9\%, en el rango de 70 a 79 años. K. pneumoniae, con un $6,4 \%$ y $4,3 \%$, en los rangos entre 60 a 69 años y 70 a 79 años, respectivamente. P. aeruginosa, se evidenció con una frecuencia de $4,3 \%$, en el rango de 70 a 79 años. P. mirabilis, con un $3,5 \%$, en el rango de 50 a 59 años.

De los 141 casos estudiados en los cuatro hospitales, objeto de estudio, se determinó que $113(80,1 \%)$ se registraron durante los primeros diez días. En el 12,1\%, representado por 17 casos, presentaron el cateterismo, durante el intervalo comprendido entre 11-20 días. En tercer lugar, se encuentra el intervalo de 31-40 días, con cinco casos que, a su vez, representan el $3,6 \%$, del total de los casos, seguido por el intervalo que corresponde a 21-30 días, con cuatro casos, correspondientes al 2,8\%, del total de las historias y, por último, se hallan los intervalos de 41-50 días y 51-60 días, cada uno con un caso, que representa el $0,7 \%$, del total evaluado. El tiempo mínimo de duración del catéter fue de un día y el tiempo máximo correspondió a 60 días. Posteriormente, se estableció la relación entre los microorganismos y la duración del cateterismo, demostrándose que la infección se mostró en los primeros diez días, luego del cateterismo, con un $32 \%$, para E. coli y $15 \%$, para K. pneumoniae. Se determinó que la infección se exteriorizó con una frecuencia menor al $1 \%$ en las pacientes que tienen cateterismo uretral, por más de 20 días.

De la muestra estudiada, la media de estancia en las Unidades de Cuidados Intensivos fue de 13,9 días y la mediana de ocho días, con una estancia mínima de dos días y una máxima de 105 días. De esta manera, la infección se presentó con un $68 \%$, en los primeros diez días. Se evidencia heterogeneidad en la muestra en cuanto al tiempo de estancia en Unidades de cuidados intensivos. E. coli, como el microorganismo más prevalente en los cultivos de las pacientes evaluadas, muestra su mayor aparición en los primeros diez días de hospitalización, con un porcentaje $28,4 \%$ y se evidencia una disminución paulatina en su progreso con el paso del tiempo. Siguiendo en orden descendente, se demostró $K$. pneumoniae, como el patógeno más prevalente en los primeros diez días de estancia en $\mathrm{UCl}$, con un 13,5\%, también con una disminución en su presentación con el paso de los días. Los resultados demuestran que la infección se genera con menor frecuencia, según la mayor estancia en UCI.

Agradecimientos. Los autores agradecen la colaboración prestada a los diferentes hospitales, directivos y funcionarios y a la Universidad de Ciencias Aplicadas y Ambientales U.D.C.A, para el desarrollo de la presente investigación. Financiación: El trabajo fue financiado por los autores del presente trabajo. Conflictos de interés: Los autores declaramos que no existen conflictos de intereses que pongan en riesgo la validez de los resultados presentados.

\section{BIBLIOGRAFÍA}

1. ÁlVAREZ, F.; PALOMAR, M.; OLAECHEA, P.; OTAL, J.J.; INSAUSTI, J.; CERDÁ, E. 2007. Estudio Nacional de Vigilancia de Infección Nosocomial en Unidades de Cuidados Intensivos: Informe evolutivo de los años 2003-2005. Med. Intensiva. 31(1):6-17.

2. AMIN, M.; MEHDINEJAD, M.; POURDANGCHI, Z. 2009. Study of bacteria isolated from urinary tract infections and determination of their susceptibility to antibiotics. Jundishapur J. Microbiol. 2(3):118-123. 
3. CHUNG, M.; LIU, C.; WOUNG, L.; LEE, W.; JENG, H.; LU, S. 2008. Species and antimicrobial resistance of uropathogens isolated from patients with urinary catheter. Curr. Probl. Pediatr. Adolesc. Health Care. 38(10):309-327.

4. DE OLIVEIRA, A.; TASSONE, C.; SOUZA, R. 2010. Nosocomial Infection in an Intensive Care Unit in a Brazilian University Hospital. Rev. Latino-Am. Enfermagem. 18(2):233-239.

5. ECHEVARRÍA, J.; AGUILAR, E.; OSORES, F. 2006. Infección del tracto urinario y manejo antibiótico. Acta Medica Per. 23(1):26-31.

6. EIROS, J.; OCHOA, C. 2007. Perfil etiológico de las infecciones urinarias y patrón de sensibilidad de los uropatógenos. An. Pediatr. 67(5):461-468.

7. ELPERN, E.; KILLEEN, K.; KETCHEM, A.; WILEY, A.; PATEL, G.; LATEEF, O. 2009. Reducing use of indwelling urinary catheters and associated urinary tract infections. Am. J. Crit. Care. 18(6):535-541.

8. GONZÁLES CAMARENA, D.E.; JAULIS SOLORZANO, J.F., TAPIA EGOAVIL, E.Z.; SAMALVIDES CUBA, F. 2008. Sensibilidad antibiótica de bacterias causantes de infecciones del tracto urinario en un hospital general. Rev Med Hered. 20(1):11-15.

9. HORAN, T.; ANDRUS, M.; DUDECK, M. 2008. CDC/ NHSN surveillance definition of health care-associated infection and criteria for specific types of infections in the acute care settin. Am. J. Infect. Control. 36(5):309-332.

10. MINDY, T.; JOYCE, L.; WEE, I. HELEN, O. 2010. Evaluation of intensive care unit-acquired urinary tract infections in Singapore. Ann. Acad. Med. Singapore. 39:460-465.

11. OCHOA, S.; EIROS, C.; PÉREZ, L. 2005. Etiología de las infecciones del tracto urinario y sensibilidad de los uropatógenos a los antimicrobianos. Rev. Esp. Quimioterap. 18(2):124-135.

12. PAVANELLO, S.; FROTA, M.; ROMERO, A.; SOARES DA SILVA, A.; MALACCHIA, J.; CAMPOS, C.; FERREIRA,
R.; DE ALMEIDA, S. 2009. Enfermería Global, Febrero 2009, No15. Disponible desde Internet en: http:// revistas.um.es/eglobal/article/view/49561/48851 (con acceso 29/03/10).

13. RAO, S.; LIN, X.; RAO, D.; YU, H. 2011. Flora distribution and drug resistance in catheter-associated urinary tract infection Int. J. Urolog. Nursing. 5:31-33.

14. ROSENTHAL, V.; MAKI, D.; MULITRAT, S.; MEDEIROS, E.; KUMAR, S.; YEPES, D.; LEBLEBICIOGLU, H.; NOVALES, M.; BERBA, R.; WONG, F.; PINO, A.; DUEÑAS, L. 2010. Informe de la Comunidad Científica Internacional de Control de Infecciones Nosocomiales, datos correspondientes al período 2003-2008, a junio de 2009; Am J Infect Control. 38(2):95-104.

15. SADER, H. 2002. Resistencia antimicrobiana en Latinoamérica. ¿Cómo estamos? Rev. Chil. Infectol. 19:S5S13.

16. SALVATIERRA, R.; BENGUIGUI, Y. 2000. Resistencia antimicrobiana en las Américas: Magnitud del problema y su contención. Organización Panamericana de la Salud. p.220-227.

17. TALAAT, M.; HAFEZ, S.; SAIED, T. 2010. Surveillance of catheterassociated urinary tract infection in 4 intensive care units at Alexandria university hospitals in Egypt. Am. J. Infect. Control. 38(3):222-228.

18. VERHAZ, A.; SKRBIĆ, R.; RAKITA, M. 2005. Resistance of catheter-associated urinary tract infections to antibacterials. Vojnosanitetski Pregled. Milit.-Med. Pharmac. Rev. 62(3):181-187.

19. WILLEY, J.; SHERWOOD L.; WOOLVERTON, C. 2009. Microbiología de Prescott, Harley y Kleim. 2009. Editorial Mc Graw Hill, 7a ed. p.835-853.

20. XIE, D.; LAI, R.; NIE, S. 2011. Surveys of catheter-associated urinary tract infection in a university hospital intensive care unit in China. Braz. J. Infect. Dis. 15(3):296-297.

Recibido: Enero 16 de 2012

Aceptado: Mayo 14 de 2012 